Bull. Korean Math. Soc. 52 (2015), No. 2, pp. 367-376

http://dx.doi.org/10.4134/BKMS.2015.52.2.367

\title{
FINITE $p$-GROUPS WHOSE NON-CENTRAL CYCLIC SUBGROUPS HAVE CYCLIC QUOTIENT GROUPS IN THEIR CENTRALIZERS
}

\author{
Lihua Zhang, Jiao Wang, and Haipeng QU \\ AbSTRACT. In this paper, we classified finite $p$-groups $G$ such that \\ $C_{G}(x) /\langle x\rangle$ \\ is cyclic for all non-central elements $x \in G$. This solved a problem pro- \\ posed By Y. Berkovoch.
}

\section{Introduction}

If $G$ is a finite group, then $1 \leq\langle x\rangle \leq C_{G}(x) \leq G$ for all elements $x \in G$. In particular, $G$ is abelian if and only if $\left|G: C_{G}(x)\right|=1$ for all $x \in G$. Moreover, K. Ishikawa [5] classified finite $p$-groups with $\left|G: C_{G}(x)\right| \leq p^{2}$. Along another line, X. H. Li and J. Q. Zhang [6] classified finite $p$-groups with $\left|C_{G}(x):\langle x\rangle\right| \leq p^{k}$, where $k=1,2$ and $p>2$. Y. Berkovich [1] proposed the following:

Problem 116(ii). Classify the $p$-groups $G$ such that $C_{G}(H) / H$ is cyclic for all noncentral cyclic $H<G$.

In other words, Problem 116(ii) requires to classify finite $p$-groups $G$ such that $C_{G}(x) /\langle x\rangle$ is cyclic for all non-central elements $x \in G$.

For convenience, the groups in Problem 116(ii) are called $\mathcal{P}$-groups. Let $\mathcal{S}=\{G \mid G$ is a $\mathcal{P}$-group $\}$. In this paper, $\mathcal{S}$ is determined, and hence the Problem 116(ii) is solved.

\section{Preliminaries}

Assume $G$ is a finite $p$-group. Let $r(G)=\max \left\{\log _{p}|E| \mid E \leq G\right.$ and $E$ is elementary abelian $\}$ and $r_{n}(G)=\max \left\{\log _{p}|E| \mid E \unlhd G\right.$ and $E$ is elementary

Received April 9, 2013; Revised September 2, 2013.

2010 Mathematics Subject Classification. 20D15.

Key words and phrases. centralizers, non-central elements, normal rank, p-groups of maximal class.

This work was supported by NSFC (no.11371232, 11471198), by NSF of Shanxi Province (no.2013011001) and FRF for the Central Univ.(Grant No.BUPT2013RC0901). 
abelian $\}. r(G)$ is called the rank of $G$ and $r_{n}(G)$ is called the normal rank of $G$.

Let $G$ be a finite $p$-group. We use $C_{p^{m}}, C_{p^{m}}^{n}$ and $H * K$ to denote the cyclic group of order $p^{m}$, the direct product of $n$ cyclic groups of order $p^{m}$, and a central product of $H$ and $K$, respectively. $M<\cdot G$ means $M$ is a maximal subgroup of $G$.

We use $\mathrm{M}_{p}(m, n)$ to denote the group

$$
\left\langle a, b \mid a^{p^{m}}=b^{p^{n}}=1, a^{b}=a^{1+p^{m-1}}\right\rangle \text {, where } m \geq 2,
$$

and $\mathrm{M}_{p}(m, n, 1)$ to denote the group

$$
\left\langle a, b, c \mid a^{p^{m}}=b^{p^{n}}=c^{p}=1,[a, b]=c,[c, a]=[c, b]=1\right\rangle,
$$

where $m \geq n$, and $m+n \geq 3$ if $p=2$. For other notation and terminology the reader is referred to [4].

A non-abelian group $G$ is said to be minimal non-abelian if every proper subgroup of $G$ is abelian. A concept which is more general than that of minimal non-abelian $p$-groups was introduced by Y. Berkovich and Z. Janko in [2]. For a positive integer $t$, a finite $p$-group $G$ is said to be an $\mathcal{A}_{t}$-group if all subgroups of index $p^{t}$ of $G$ are abelian, and at least one subgroup of index $p^{t-1}$ of $G$ is not abelian. Obviously, $\mathcal{A}_{1}$-groups are exactly the minimal non-abelian $p$-groups.

Lemma 2.1 ([7, Lemma 2.2]). Suppose that $G$ is a finite nonabelian p-group. Then the following conditions are equivalent.

(1) $G$ is minimal nonabelian;

(2) $d(G)=2$ and $\left|G^{\prime}\right|=p$;

(3) $d(G)=2$ and $\Phi(G)=Z(G)$.

Lemma 2.2 ([1, Proposition 72.1]). Assume $G$ is a metacyclic p-group. Then $G$ is an $\mathcal{A}_{t}$-group if and only if $\left|G^{\prime}\right|=p^{t}$.

Lemma 2.3. Assume $G$ is a finite p-group and $c(G)=2$. Then $G^{\prime}$ is elementary abelian if and only if $G / Z(G)$ is elementary abelian.

Proof. It follows from $\left[a^{p}, b\right]=1 \Longleftrightarrow[a, b]^{p}=1$ for all $a, b \in G$.

Lemma 2.4 ([1, Section 1, Exercise 69(a)]). Assume $G$ is a finite p-group. If two distinct maximal subgroups of $G$ are abelian, then $\left|G^{\prime}\right| \leq p$.

Lemma 2.5 ([1, Theorem 41.1]). If $G$ is a minimal non-metacyclic 2-group, then $|G| \leq 2^{5}$.

Lemma 2.6 ([3, Theorem 4.1]). Assume $G$ is a group of order $p^{n}$ with $p>2$ and $n \geq 5$. If $r_{n}(G)=2$, then $G$ is one of the following groups.

(1) $G$ is metacyclic;

(2) $G \cong \mathrm{M}_{p}(1,1,1) * C_{p^{n-2}}$;

(3) $G$ is a 3-group of maximal class of order $\geq 3^{5}$;

(4) $G=\left\langle a, x, y \mid a^{p^{n-2}}=1, x^{p}=y^{p}=1,[a, x]=y,[x, y]=a^{i p^{n-3}},[y, a]=1\right\rangle$, $i=1$ or $\sigma$, where $\sigma$ is a fixed square non-residue modulo $p$. 
Lemma 2.7 ([1, Theorem 9.10]). If a group $G$ of order $p^{m}>p^{3}$ has a subgroup $M$ of order $p^{m-1}$ of maximal class, then $G$ is either of maximal class or $G / G^{\prime} \cong$ $C_{p}^{3}$.

Lemma 2.8 ([1, Section 9, Exercise $1(\mathrm{c})])$. Assume $G$ is a group of maximal class and order $p^{n}$. If $p>2$ and $n>3$, then $G$ has no cyclic normal subgroups of order $p^{2}$.

Lemma 2.9 ([1, Section 9, Exercise 10]). Let $G$ be a 3-group of maximal class. Then the fundamental subgroup $G_{1}$ of $G$ is abelian or metacyclic minimal nonabelian.

\section{Some properties of $\mathcal{P}$-groups}

Lemma 3.1. If $G$ is a $\mathcal{P}$-group, then $r(G) \leq 2$.

Proof. If not, then there exists $A \leq G$ and $A \cong C_{p}^{3}$. If $A \not \leq Z(G)$, then there exists $x \in A \backslash Z(G)$. Since $A$ is abelian, $A \leq C_{G}(x)$. Since $A /\langle x\rangle \cong C_{p}^{2}$, $C_{G}(x) /\langle x\rangle$ is not cyclic. This contradicts hypothesis. If $A \leq Z(G)$, then $A\langle x\rangle /\langle x\rangle \leq C_{G}(x) /\langle x\rangle$ for all $x \in G \backslash Z(G)$. Since $A\langle x\rangle /\langle x\rangle \cong A / A \cap\langle x\rangle \cong C_{p}^{2}$ or $C_{p}^{3}, C_{G}(x) /\langle x\rangle$ is not cyclic. This contradicts hypothesis again.

Lemma 3.2. Assume $G$ is a metacyclic nonabelian $p$-group and $p>2$. Then $G$ is a $\mathcal{P}$-group if and only if $G$ is minimal nonabelian.

Proof. $\Longleftarrow$ : Let $x \in G \backslash Z(G)$. By Lemma 2.1(3), $Z(G)=\Phi(G)$. Since $\Phi\left(C_{G}(x)\right) \leq \Phi(G), x \notin \Phi\left(C_{G}(x)\right)$. Since $G$ is metacyclic, $C_{G}(x)$ is metacyclic. So $d\left(C_{G}(x)\right) \leq 2$. It follows that there exists $y \in G$ such that $C_{G}(x)=\langle x, y\rangle$. Hence $C_{G}(x) /\langle x\rangle$ is cyclic. That is, $G$ is a $\mathcal{P}$-group.

$\Longrightarrow$ : Since $p>2$ and $G$ is metacyclic, $\Omega_{1}(G) \cong C_{p}^{2}$. Let $G=\langle a, b\rangle$ and $H=\langle a\rangle \Omega_{1}(G)$, where $\langle a\rangle \triangleleft G$. Then $H^{\prime} \leq\langle a\rangle \cap \Omega_{1}(G)$. In particular, $\left|H^{\prime}\right| \leq p$. Thus $\left\langle a^{p}\right\rangle \Omega_{1}(G)$ is abelian. Hence $H \leq C_{G}\left(a^{p}\right)$. Since $G$ is a $\mathcal{P}$-group and $H /\left\langle a^{p}\right\rangle=H / \mho_{1}(H) \cong C_{p}^{2}$, we get $a^{p} \in \bar{Z}(G)$. Since $p>2$ and $G$ is metacyclic, $G$ is regular. Hence $[a, b]^{p}=1$ is equivalent to $\left[a^{p}, b\right]=1$. It follows that $\left|G^{\prime}\right|=p$. By Lemma 2.1(2), $G$ is minimal nonabelian.

It is easy to see that the argument in Lemma 3.2 is true for ordinary metacyclic 2-groups. Thus we have:

Corollary 3.3. Assume $G$ is an ordinary metacyclic 2-group. Then $G$ is a $\mathcal{P}$-group if and only if $G$ is minimal nonabelian.

Lemma 3.4. Assume $G$ is a $\mathcal{P}$-group and $H$ is a nonabelian subgroup of $G$. Then $H$ is a $\mathcal{P}$-group.

Proof. $\forall x \in H \backslash Z(H)$, we have $x \notin Z(G)$. If not, $x \in Z(G) \cap H \leq Z(H)$, a contradiction. Thus $x \in G \backslash Z(G)$. Since $G$ is a $\mathcal{P}$-group, $C_{G}(x) /\langle x\rangle$ is cyclic. Since $C_{H}(x) \leq C_{G}(x), C_{H}(x) /\langle x\rangle$ is cyclic. 


\section{4. $\mathcal{P}$-groups of order odd}

Theorem 4.1. Let $p$ be an odd prime. Then $G$ is a $\mathcal{P}$-group if and only if $G$ is one of the following pairwise non-isomorphic groups.

(1) metacyclic minimal nonabelian p-groups of order $p^{n}$, where $n>3$;

(2) $\mathrm{M}_{p}(1,1,1)$;

(3) $G=\left\langle a, b, c \mid a^{9}=c^{3}=1, b^{3}=a^{3},[a, b]=c,[c, a]=1,[c, b]=a^{-3}\right\rangle$;

(4) $G \cong \mathrm{M}_{p}(1,1,1) * C_{p^{n-2}}$, where $n>2$;

(5) $G=\left\langle a, x, y \mid a^{p^{n-2}}=1, x^{p}=y^{p}=1,[a, x]=y,[x, y]=a^{i p^{n-3}},[y, a]=1\right\rangle$, $i=1$ or $\sigma$, where $\sigma$ is a fixed square non-residue modulo $p$.

Proof. If $|G| \leq p^{4}$, then, the conclusion holds by checking the list of groups of order $p^{3}$ and $p^{4}$. Assume $|G| \geq p^{5}$. By Lemma 3.1, $r(G) \leq 2$. Thus $r_{n}(G) \leq 2$. If $r_{n}(G)=1$, then $G$ is cyclic, a contradiction. So $r_{n}(G)=2$. Thus $G$ is one of the groups listed in Lemma 2.6. We discuss case by case.

If $G$ is the group (1) in Lemma 2.6, then, by Lemma 3.2, $G$ is the group (1).

If $G$ is the group (2) in Lemma 2.6, then $Z(G)$ is a cyclic subgroup of index $p^{2}$. Let $g \in G \backslash Z(G)$. Then $C_{G}(g)<G$. Obviously, $C_{G}(g) \geq\langle g\rangle Z(G)$. Thus $C_{G}(g)=\langle g\rangle Z(G)$. Hence $C_{G}(g) /\langle g\rangle \cong Z(G) / Z(G) \cap\langle g\rangle$ is cyclic. That is, $G$ is a $\mathcal{P}$-group. This is the group (4).

If $G$ is the group (3) in Lemma 2.6, then, by Lemma $2.9, G_{1}$ is abelian or metacyclic non-abelian. Since $|G| \geq 3^{5},\left|G_{1}\right| \geq 3^{4}$. It follows that there exists a subgroup $H \unlhd G$ such that $|H|=3^{4}$ with $d(H) \geq 2$ and $H$ is abelian or metacyclic non-abelian. By Lemma 3.1, $d(H)=2$. By Lemma 2.8, $\mho_{1}(H) \not$ $C_{9}$. Hence $H \cong C_{9}^{2}$ or $\mathrm{M}_{3}(2,2)$. Thus $\mho_{1}(H) \leq Z(H)$ and $\mho_{1}(H) \cong C_{3}^{2}$. Since $G$ is of maximal class, $|Z(G)|=3$. So there exists $x \in \mho_{1}(H) \backslash Z(G)$. Since $H$ is not cyclic, $H /\langle x\rangle$ is not cyclic. It follows from $H \leq C_{G}(x)$ that $C_{G}(x) /\langle x\rangle$ is not cyclic. That means $G$ is not a $\mathcal{P}$-group.

If $G$ is the group (4) in Lemma 2.6, then, by calculations, we get $Z(G)=\left\langle a^{p}\right\rangle$ is a subgroup of index $p^{3}$ and $\langle a, y\rangle$ is a abelian maximal subgroup. Since $\left|G^{\prime}\right|=p^{2}, G$ has a unique abelian maximal subgroup by Lemma 2.4. Let $g \in G \backslash Z(G)$. Then $\left\langle a^{p}, g\right\rangle \leq C_{G}(g)<G$. If $C_{G}(g)=\left\langle a^{p}, g\right\rangle$, then $C_{G}(g) /\langle g\rangle$ is cyclic. If $C_{G}(g)>\left\langle a^{p}, g\right\rangle$, then $C_{G}(g) \lessdot G$. Since $\left\langle a^{p}, g\right\rangle \leq Z\left(C_{G}(g)\right)$ and $\left|C_{G}(g) /\left\langle a^{p}, g\right\rangle\right|=p, C_{G}(g)$ is abelian. Thus $C_{G}(g)=\langle a, y\rangle$ and $g=a^{i} y^{j}$, where $(i, p)=1$ or $(j, p)=1$. It follows that $C_{G}(g) /\langle g\rangle$ is cyclic. Hence $G$ is a $\mathcal{P}$-group. This is the group (5).

\section{5. $\mathcal{P}$-groups of order even}

Lemma 5.1. Assume $G$ is a $\mathcal{P}$-group of order $2^{n}$ and $n \geq 5, M$ is a maximal subgroup of $G$. If $M$ is of maximal class, then $G$ is of maximal class.

Proof. Otherwise, by Lemma $2.7, G / G^{\prime} \cong C_{p}^{3}$. It follows that

$$
\mho_{1}(G)=G^{\prime}=M^{\prime}=\mho_{1}(M)
$$


and hence $\exp (G)=\exp (M)$. Since $M$ is of maximal class, $M$ has a maximal subgroup $H$ which is cyclic by the classification of 2-groups of maximal class. Let $K=\Omega_{2}(H)$. Then $K \cong C_{4}$ and $K$ char $H$ char $M \unlhd G$. Hence $K \unlhd G$. By $N / C$ theorem,

$$
G / C_{G}(K) \lesssim \operatorname{Aut}(K) \cong C_{2} .
$$

Since $M$ is of maximal class, $|Z(M)|=2$. Hence $K \not \leq Z(M)$. Thus $G / C_{G}(K) \cong$ $C_{2}$. Since $G$ is not of maximal class, $C_{G}(K)$ is not cyclic. Since $n \geq 5$,

$$
K \leq \mho_{1}\left(M^{\prime}\right) \leq \Phi\left(C_{G}(K)\right) .
$$

It follows that $C_{G}(K) / K$ is not cyclic. This contradicts that $G$ is a $\mathcal{P}$-group.

Lemma 5.2. Assume $G$ is a $\mathcal{P}$-group of order $2^{n}$ and $n \geq 6, M$ is a maximal subgroup of $G$ and $M=\langle a, b, c| a^{2^{n-4}}=b^{2^{2}}=1, c^{2}=a^{2} b^{2},[a, b]=b^{2},[c, a]=$ $[c, b]=1\rangle$. Then

(1) $Z(M)=\left\langle a^{2}, c\right\rangle=\left\langle b^{2}, c\right\rangle \cong C_{2} \times C_{2^{n-4}}$ and $\Omega_{1}(M)=\left\langle a^{2^{n-5}}, b^{2}\right\rangle \cong C_{2}^{2}$;

(2) $\Phi(G)=\mho_{1}(G) \leq Z(M) \leq Z(G)$;

(3) $G^{\prime} \leq \Omega_{1}(Z(M))=\Omega_{1}(M)$;

(4) $M=\Omega_{n-4}(G)$, in particular, o(x)=2 $2^{n-3}$ for all $x \in G \backslash M$.

Proof. (1) Obviously.

(2) Firstly, we prove $Z(M) \leq Z(G)$. If $a^{2} \notin Z(G)$, then $C_{G}\left(a^{2}\right) /\left\langle a^{2}\right\rangle$ is cyclic since $G$ is a $\mathcal{P}$-group. Since $C_{G}\left(a^{2}\right) \geq M, M /\left\langle a^{2}\right\rangle$ is cyclic. This is a contradiction. Thus $a^{2} \in Z(G)$. Similarly, $c \in Z(G)$.

Secondly, we prove $x^{2} \in Z(M)$ for all $x \in G$. Since $x^{2} \in M$, it suffices to prove $x^{2} \in Z(G)$. If not, then, since $C_{G}\left(x^{2}\right) /\left\langle x^{2}\right\rangle \geq Z(M)\langle x\rangle /\left\langle x^{2}\right\rangle$ and $G$ is a $\mathcal{P}$-group, $Z(M)\langle x\rangle /\left\langle x^{2}\right\rangle$ is cyclic. Since $\left\langle x^{2}\right\rangle \leq \Phi(Z(M)\langle x\rangle), Z(M)\langle x\rangle$ is cyclic. In particular, $Z(M)$ is cyclic. This is a contradiction.

(3) By $(2), G / Z(G)$ is elementary abelian. By Lemma $2.3, G^{\prime}$ is elementary abelian. In particular, $G^{\prime} \leq \Omega_{1}(Z(M))=\Omega_{1}(M)$.

(4) If not, then $\Omega_{n-4}(G)=G$. By (2) and (3), $G$ is 4 -abelian. Since $n-4 \geq 2$, $\exp (G)=\exp \left(\Omega_{n-4}(G)\right)=2^{n-4}$. Hence $\exp \left(\mho_{1}(G)\right)=2^{n-5}$. It follows that

$$
\Phi(G)=\mho_{1}(G) \leq \Omega_{n-5}(Z(M))=\left\langle b^{2}, c^{2}\right\rangle=\Phi(M) .
$$

Thus $d(G)=d(M)+1=4$.

Take $x \in G \backslash M$. Then $\langle a, b, c, x\rangle=G$. Since

$$
G^{\prime} \leq \Omega_{1}(M)=\left\langle a^{2^{n-5}}, b^{2}\right\rangle,
$$

$[a, x] \in\left\langle a^{2^{n-5}}\right\rangle$ or $[a, b x] \in\left\langle a^{2^{n-5}}\right\rangle$. Without loss of generality, we can assume $[a, x] \in\left\langle a^{2^{n-5}}\right\rangle$. If $[a, x]=1$, then

$$
C_{G}(a) /(\langle a\rangle \Phi(G)) \geq\langle\bar{c}, \bar{x}\rangle \cong C_{2}^{2}
$$

Hence $C_{G}(a) /\langle a\rangle$ is not cyclic. This is a contradiction. So $[a, x]=a^{2^{n-5}}$.

Note that $c \in Z(G)$ and $[a, x]=\left[a, x c^{i}\right]$, where $i$ is an integer. Since

$$
x^{2} \in \Phi(G)=\left\langle b^{2}, c^{2}\right\rangle,
$$


$\left(x c^{i}\right)^{2}=c^{2}$ or $\left(x c^{i}\right)^{2}=c^{2} b^{2}=a^{2}$ for a suitable $i$. Without loss of generality, we assume $x^{2}=c^{2}$ or $a^{2}$. If $x^{2}=c^{2}$, then $o\left(x c^{-1}\right)=2$. If $x^{2}=a^{2}$, then $o\left(x a^{-1+2^{n-6}}\right)=2$. In either case, there is an involution $y \in G \backslash M$. Hence $\langle y\rangle \Omega_{1}(M) \cong C_{2}^{3}$, This contradicts Lemma 3.1.

Lemma 5.3. Assume $G$ and $M$ are the same as Lemma 5.2. Then $G$ is isomorphic to one of the following non-isomorphic groups:

(1) $\left\langle a, b, c \mid a^{2^{n-3}}=b^{2^{2}}=1, c^{2}=a^{2} b^{2},[a, b]=b^{2},[c, a]=[c, b]=1\right\rangle$;

(2) $\left\langle a, b, c \mid a^{2^{n-3}}=b^{2^{2}}=1, c^{2}=a^{2} b^{2},[a, b]=b^{2},[c, a]=a^{2^{n-4}},[c, b]=1\right\rangle$.

Proof. By Lemma 5.2(4), $\exp (G)=2^{n-3}$ and $o(x)=2^{n-3}$ for all $x \in G \backslash M$. By Lemma 5.2(2), $x^{2} \in Z(M)=\left\langle b^{2}, c\right\rangle$. We can assume $x^{2}=c$ or $x^{2}=c b^{2}$. By Lemma $5.2(3), G^{\prime} \cong C_{2}$ or $G^{\prime} \cong C_{2}^{2}$.

If $G^{\prime} \cong C_{2}$, then $G^{\prime}=M^{\prime}=\left\langle b^{2}\right\rangle$. If $[b, x]=1$ and $[a, x]=1$, then let $a_{1}=a x^{-1}$. Thus

$$
a_{1}^{2^{n-3}}=1,\left[a_{1}, b\right]=\left[a x^{-1}, b\right]=[a, b]=b^{2} \text { and } a_{1}^{2} b^{2}=x^{2} .
$$

Hence

$$
G=\left\langle a_{1}, b, x \mid a_{1}^{2^{n-3}}=b^{2^{2}}=1, x^{2}=a_{1}^{2} b^{2},\left[a_{1}, b\right]=b^{2},\left[x, a_{1}\right]=[x, b]=1\right\rangle .
$$

Here $G$ is isomorphic to the group (1). If $[b, x]=1$ and $[a, x]=b^{2}$, then let $x_{1}=b x$. Thus $\left[b, x_{1}\right]=1$ and $\left[a, x_{1}\right]=1$. If $[b, x]=b^{2}$, then let $x_{1}=a x$. Thus $\left[b, x_{1}\right]=1$. In the two cases, we also get $G$ is isomorphic to the group (1).

If $G^{\prime} \cong C_{2}^{2}$, then $G^{\prime}=\Omega_{1}(M)$. We consider the possible cases of $[b, x]$.

Case 1. $[b, x]=1$

Then $[a, x]=a^{2^{n-5}}$ or $[a, x]=a^{2^{n-5}} b^{2}$.

If $[a, x]=a^{2^{n-5}}$, then let $a_{1}=a x^{-1+2^{n-5}}$. Thus

$$
\begin{gathered}
a_{1}^{2^{n-3}}=1, x^{2}=a_{1}^{2} b^{2},\left[a_{1}, b\right]=\left[a x^{-1+2^{n-5}}, b\right]=[a, b]=b^{2}, \\
{\left[a_{1}, x\right]=\left[a x^{-1+2^{n-5}}, x\right]=[a, x]=a^{2^{n-5}}=a_{1}^{2^{n-4}} .}
\end{gathered}
$$

Thus $G=\langle a, b, x\rangle=\left\langle a_{1}, b, x\right\rangle$ with defining relations as above. Here $G$ is isomorphic to the group (2).

If $[a, x]=a^{2^{n-5}} b^{2}$, then let $x_{1}=b x$. Thus $\left[b, x_{1}\right]=1$ and $\left[a, x_{1}\right]=a^{2^{n-5}}$. We get the group (2) as that of $[a, x]=a^{2^{n-5}}$.

Case 2. $[b, x]=b^{2}$

Let $x_{1}=a x$. Then $\left[b, x_{1}\right]=1$. This is reduced to Case 1 .

Case 3. $[b, x]=a^{2^{n-5}}$

If $[a, x]=1$, then let $a_{1}=a x^{-1}, b_{1}=b x^{2^{n-5}}$ and $x_{1}=x^{1+2^{n-5}}$. By calculations, we get

$$
\begin{gathered}
o\left(a_{1}\right)=2^{n-3}, o\left(b_{1}\right)=2^{2},\left[a_{1}, b_{1}\right]=b^{2} x^{2^{n-4}}=b_{1}^{2}, \\
{\left[a_{1}, x_{1}\right]=1,\left[b_{1}, x_{1}\right]=x_{1}^{2^{n-4}}, a_{1}^{2} b_{1}^{2}=x_{1}^{2} .}
\end{gathered}
$$


Thus $G=\langle a, b, x\rangle=\left\langle a_{1}, b_{1}, x_{1}\right\rangle$ with defining relations as above. By a simple checking we get $G$ is isomorphic to the group (2).

If $[a, x]=b^{2}$, then let $a_{1}=a$ and $x_{1}=b x$. If $[a, x]=a^{2^{n-5}}$, then let $a_{1}=a b$ and $x_{1}=x$. If $[a, x]=a^{2^{n-5}} b^{2}$, then let $a_{1}=a b$ and $x_{1}=b x$. In this three cases, we get $\left[a_{1}, x_{1}\right]=1$ and $\left[b, x_{1}\right]=a_{1}^{2^{n-5}}$. This is reduced to the case of $[a, x]=1$.

Case 4. $[b, x]=a^{2^{n-5}} b^{2}$

Let $x_{1}=a x$. Then $\left[b, x_{1}\right]=a^{2^{n-5}}$. This is reduced to Case 3 .

Lemma 5.4. Assume $G$ is a $\mathcal{P}$-group of order $2^{n}$ and $n \geq 6, M$ is a maximal subgroup of $G$ and $M=\langle a, b, c| a^{2^{n-4}}=b^{2^{2}}=1, c^{2}=a^{2} b^{2},[a, b]=b^{2},[c, a]=$ $\left.a^{2^{n-5}},[c, b]=1\right\rangle$. Then $n=6$ and $G \cong\langle a, b, c, d| a^{4}=b^{4}=1, c^{2}=a^{2} b^{2}, b^{2}=$ $\left.d^{2},[a, b]=b^{2},[a, c]=a^{2},[a, d]=1,[b, c]=1,[b, d]=a^{2},[c, d]=c^{2}\right\rangle$.

Proof. By a similar argument as that of Lemma 5.2, we get

(1) $\Phi(M)=Z(M)=\left\langle a^{2}, b^{2}\right\rangle \cong C_{2} \times C_{2^{n-5}}$ and $\Omega_{1}(M)=M^{\prime}=\left\langle a^{2^{n-5}}, b^{2}\right\rangle$ $\cong C_{2}^{2}$

(2) $\Phi(G)=\Phi(M)=Z(M) \leq Z(G)$, in particular, $d(G)=4$ and $\exp (G)=$ $2^{n-4}$

(3) $G^{\prime}=\Omega_{1}(Z(M))=\Omega_{1}(M)=M^{\prime}$;

(4) $G \backslash M$ has no element of order 2 .

Noting $[a, M]=M^{\prime}=G^{\prime}$, we can take a suitable $d \in G \backslash M$ such that $[a, d]=1$. Then $G=\langle a, b, c, d\rangle$. Assume $d^{2}=a^{2 i} b^{2 j}$, where $i$ and $j$ are integers. Replacing $d$ by $d a^{-i}$, we can assume $d^{2}=b^{2 j}$. By (4), $j \neq 0$. Hence $d^{2}=b^{2}$.

If $[b, d] \in\left\langle b^{2}\right\rangle$, then $[b, d]=1$ or $[b, a d]=1$. Hence $\left|C_{G}(b) /(\langle b\rangle \Phi(G))\right| \geq 4$. This contradicts that $G$ is a $\mathcal{P}$-group. Thus $[b, d]=a^{2^{n-5}}$ or $a^{2^{n-5}} b^{2}$. Similarly, $[c, d]=b^{2}$ or $b^{2} a^{2^{n-5}}$.

If $n \geq 7$, then $a^{2^{n-6}} \in \mho_{1}(G) \leq Z(G)$. Hence $\left(b d a^{2^{n-6}}\right)^{2}=(b d)^{2} a^{2^{n-5}}=$ $[b, d] a^{2^{n-5}}$. By (4), $[b, d] a^{2^{n-5}} \neq 1$. Thus $[b, d]=b^{2} a^{2^{n-5}}$. It follows that $(a b c d)^{2}=b^{2}[c, d]$. By $(4), b^{2}[c, d] \neq 1$. Thus $[c, d]=b^{2} a^{2^{n-5}}=[b, d]$. So $[b c, d]=1$. It follows that $\left|C_{G}(d) /(\langle d\rangle \Phi(G))\right| \geq 4$. This contradicts that $G$ is a $\mathcal{P}$-group. Hence $n=6$.

By $(4), 1 \neq(a b d)^{2}=a^{2} b^{2}[b, d]$. Hence $[b, d]=a^{2}$. By (4) again, $1 \neq(b c d)^{2}=$ $b^{2}[c, d]$. Hence $[c, d]=b^{2} a^{2}=c^{2}$, and we get the desired group $G$.

Lemma 5.5. Assume $G$ is a $\mathcal{P}$-group of order $2^{n}$ and $n \geq 5, M$ is a maximal subgroup of $G$ and $M=\langle a, b, c| a^{2^{n-3}}=b^{2}=c^{2}=1,[c, b]=a^{2^{n-4}},[b, a]=$ $[c, a]=1\rangle \cong D_{8} * C_{2^{n-3}}$. Then $G \cong D_{8} * C_{2^{n-2}}$.

Proof. By a similar and more simple argument as that of Lemma 5.2, we get

(1) $Z(M)=\langle a\rangle \cong C_{2^{n-3}}$ and $\Omega_{1}(Z(M))=\left\langle a^{2^{n-4}}\right\rangle$;

(2) $\Phi(G)=\mho_{1}(G) \leq Z(M) \leq Z(G)$, in particular, $\exp (G) \leq 2^{n-2}$; 
(3) $G^{\prime}=\Omega_{1}(Z(M))=M^{\prime}$;

(4) $M=\Omega_{n-3}(G)$, in particular, $o(x)=2^{n-2}$ for all $x \in G \backslash M$.

By (4), we can assume $x^{2}=a$. We consider $[b, x]$ and $[c, x]$. If $[b, x]=1$ and $[c, x]=1$, then $G=\langle b, c\rangle *\langle x\rangle \cong D_{8} * C_{2^{n-2}}$. If $[b, x]=1$ and $[c, x]=a^{2^{n-4}}$, then, by letting $x_{1}=b x$, we get $\left[b, x_{1}\right]=1$ and $\left[c, x_{1}\right]=[c, b x]=1$. Thus $G=\langle b, c\rangle *\left\langle x_{1}\right\rangle \cong D_{8} * C_{2^{n-2}}$. If $[b, x]=a^{2^{n-4}}$, then, by letting $x_{1}=c x$, we get $\left[b, x_{1}\right]=1$. This is reduced to that of $[b, x]=1$.

Lemma 5.6. Assume $G$ is a $\mathcal{P}$-group of order $2^{6}$. Then $G$ has no subgroup $M \cong\left\langle a, b, c \mid a^{4}=c^{4}=1, a^{2}=b^{2},[a, b]=a^{2},[c, a]=c^{2},[c, b]=1\right\rangle$.

Proof. Otherwise, by a similar argument as that of Lemma 5.2, we get

(1) $\Phi(M)=Z(M)=\Omega_{1}(M)=M^{\prime}=\left\langle a^{2}, c^{2}\right\rangle \cong C_{2}^{2}$;

(2) $\Phi(G)=\Phi(M)=Z(M) \leq Z(G)$, in particular, $d(G)=4$ and $\exp (G)=4$;

(3) $G^{\prime}=\Omega_{1}(Z(M))=\Omega_{1}(M)=M^{\prime}$;

(4) $G \backslash M$ has no element of order 2 .

Notice that $[a, M]=M^{\prime}=G^{\prime}$. We can take a suitable $x \in G \backslash M$ such that $[a, x]=1$. Without loss of generality, we can assume $x^{2}=c^{2}$. By a similar argument as that of Lemma 5.4 , we have $[b, x]=c^{2}$ or $a^{2} c^{2}$ and $[c, x]=a^{2}$ or $a^{2} c^{2}$.

By $(4), 1 \neq(a b x)^{2}=a^{2} c^{2}[b, x]$ and $1 \neq(a c x)^{2}=a^{2} c^{2}[c, x]$. Hence $[b, x]=c^{2}$ and $[c, x]=a^{2}$. It follows that $(a b c x)^{2}=1$. This contradicts (4).

Lemma 5.7. Assume $G$ is a $\mathcal{P}$-group of order $2^{7}$. Then $G$ has no subgroup $M \cong\langle a, b, c, d| a^{4}=b^{4}=1, c^{2}=a^{2} b^{2}, b^{2}=d^{2},[a, b]=b^{2},[a, c]=a^{2},[a, d]=$ $\left.1,[b, c]=1,[b, d]=a^{2},[c, d]=c^{2}\right\rangle$.

Proof. Otherwise, by a similar argument as that of Lemma 5.2, we get

(1) $\Phi(M)=Z(M)=\Omega_{1}(M)=M^{\prime}=\left\langle a^{2}, b^{2}\right\rangle \cong C_{2}^{2}$;

(2) $\Phi(G)=\Phi(M)=Z(M) \leq Z(G)$, in particular, $d(G)=5$ and $\exp (G)=4$;

(3) $G^{\prime}=\Omega_{1}(Z(M))=\Omega_{1}(M)=M^{\prime}$;

Notice that $[a, M]=M^{\prime}=G^{\prime}$. We can take a suitable $x \in G \backslash M$ such that $[a, x]=1$. Hence $C_{G}(a) /(\langle a\rangle \Phi(G)) \geq\langle\bar{d}, \bar{x}\rangle \cong C_{2}^{2}$. This contradicts that $G$ is a $\mathcal{P}$-group.

Theorem 5.8. Assume $G$ is a group of order $2^{n}$. Then $G$ is a $\mathcal{P}$-group if and only if $G$ is one of the following pairwise non-isomorphic groups.

(1) metacyclic minimal nonabelian p-groups;

(2) 2-groups of maximal class;

(3) $D_{8} * C_{2^{n-2}}$;

(4) $\left\langle a, b, c \mid a^{2^{n-3}}=b^{2^{2}}=1, c^{2}=a^{2} b^{2},[a, b]=b^{2},[c, a]=[c, b]=1\right\rangle$;

(5) $\left\langle a, b, c \mid a^{2^{n-3}}=b^{2^{2}}=1, c^{2}=a^{2} b^{2},[a, b]=b^{2},[c, a]=a^{2^{n-4}},[c, b]=1\right\rangle$;

(6) $\mathrm{Q}_{8} \times \mathrm{C}_{2}$;

(7) $\left\langle a, b, c \mid a^{4}=c^{4}=1, a^{2}=b^{2},[a, b]=a^{2},[c, a]=c^{2},[c, b]=1\right\rangle$; 
(8) $\langle a, b, c, d| a^{4}=b^{4}=1, c^{2}=a^{2} b^{2}, b^{2}=d^{2},[a, b]=b^{2},[a, c]=a^{2},[a, d]=$ $\left.1,[b, c]=1,[b, d]=a^{2},[c, d]=c^{2}\right\rangle$.

Proof. If $n \leq 5$, then, by classification of 2-groups of order $\leq 2^{5}$, the conclusion holds. In following assume $n \geq 6$ and $G$ is a $\mathcal{P}$-group.

By induction hypothesis, each maximal subgroup of $G$ is abelian or isomorphic to one of the groups (1)-(5), (7) and (8). If $G$ has a maximal subgroup which is isomorphic to one of the groups (2)-(5), (7) and (8), then $G$ is isomorphic to one of the groups (2)-(5) and (8) by Lemma 5.1, Lemmas 5.3, 5.4, 5.5, 5.6 and 5.7 .

Assume every maximal subgroup of $G$ is abelian or metacyclic minimal nonabelian. By Lemma 3.1, every maximal subgroup of $G$ is metacyclic. If $G$ is not metacylcic, then $G$ is minimal non-metacyclic. It follows that $|G| \leq 2^{5}$ by Lemma 2.5. This contradicts $|G| \geq 2^{6}$. Thus $G$ is metacyclic.

If $G$ is minimal non-abelian, then we get the group (1).

If $G$ is not minimal nonabelian, then $G$ is a metacyclic $\mathcal{A}_{2}$-group. By Lemma 2.2, $\left|G^{\prime}\right|=4$. Assume $G=\langle a, b\rangle$, where $G^{\prime}<\langle a\rangle$. Then $o(a) \geq 8$ and $a^{t} \in Z(G)$ if and only if $4 \mid t$. Hence $a^{2} \notin Z(G)$. Since $|G| \geq 2^{6}$ and $\left|G^{\prime}\right|=4, G$ has no cyclic maximal subgroup. It follows that $C_{G}\left(a^{2}\right)=\left\langle a, b^{2}\right\rangle$ is not cyclic. Notice that $\left\langle a^{2}\right\rangle \leq \mho_{1}\left(C_{G}\left(a^{2}\right)\right)$. $C_{G}\left(a^{2}\right) /\left\langle a^{2}\right\rangle$ is not cyclic. This contradicts $G$ is a $\mathcal{P}$-group.

It is easy to see that those groups in the theorem are pairwise non-isomorphic. In following we prove those groups in the theorem are $\mathcal{P}$-groups.

If $G$ is the group (1), then $G$ is a $\mathcal{P}$-group by Corollary 3.3 .

If $G$ is the group (2), then $G$ has a cyclic subgroup of index 2 and $G$ is metacyclic by the classification of 2-groups of maximal class. Let $\langle a\rangle$ be a cyclic subgroup of index 2 of $G$. Then $\Phi(G)=\left\langle a^{2}\right\rangle$ and $Z(G)=\left\langle a^{2^{n-2}}\right\rangle$. Let $x \in G \backslash Z(G)$. If $x \notin \Phi(G)$, then $x \notin \Phi\left(C_{G}(x)\right)$. Since $G$ is metacyclic, $C_{G}(x)$ is metacyclic. Hence $d\left(C_{G}(x)\right) \leq 2$. Thus there exists $y \in G$ such that $C_{G}(x)=\langle x, y\rangle$. It follows that $C_{G}(x) /\langle x\rangle=\langle\bar{y}\rangle$ is cyclic. If $x \in \Phi(G) \backslash Z(G)$, then $C_{G}(x)=\langle a\rangle$. Obviously, $C_{G}(x) /\langle x\rangle$ is cyclic. So $G$ is a $\mathcal{P}$-group.

If $G$ is one of the groups (3)-(7), then $|G: Z(G)| \leq 8$. It follows that $|G:\langle x, Z(G)\rangle| \leq 4$ for all $x \in G \backslash Z(G)$. Notice that $\langle x, Z(G)\rangle \leq Z\left(C_{G}(x)\right)$ and $C_{G}(x)<G$. We have $\left|C_{G}(x) / Z\left(C_{G}(x)\right)\right| \leq 2$. Hence $C_{G}(x)$ is abelian. It is easy to check $r(G)=2$. Hence $d\left(C_{G}(x)\right) \leq 2$. Thus there exists $y \in G$ such that $C_{G}(x)=\langle x, y\rangle$. It follows that $C_{G}(x) /\langle x\rangle=\langle\bar{y}\rangle$ is cyclic.

If $G$ is the group (8), then $Z(G)=\Phi(G)=\Omega_{1}(G) \cong C_{2}^{2}$. It is easy to check $Z(M)=Z(G)$ for all subgroups $M$ of order 32 . Since $Z\left(C_{G}(x)\right) \geq\langle x, Z(G)\rangle$ for all $x \in G \backslash M, Z\left(C_{G}(x)\right)>Z(G)$. Thus $\left|C_{G}(x)\right| \leq 16$. It follows that $C_{G}(x)$ is abelian and hence $d\left(C_{G}(x)\right) \leq 2$. Thus there exists $y \in G$ such that $C_{G}(x)=\langle x, y\rangle$. It follows that $C_{G}(x) /\langle x\rangle=\langle\bar{y}\rangle$ is cyclic.

Acknowledgments. We cordially thank the referee for valuable comments. All these helped us to improve the whole paper substantially. 


\section{References}

[1] Y. Berkovich, Groups of Prime Power Order, Volume 1, Walter de Gruyter, Berlin, 2008.

[2] Y. Berkovich and Z. Janko, Structure of finite p-groups with given subgroups, Contemp. Math. 402, 13-93, Amer. Math. Soc., Providence, RI, 2006.

[3] N. Blackburn, Generalizations of certain elementary theorems on p-groups, Proc. London Math. Soc. (3) 11 (1961), 1-22.

[4] B. Huppert, Endliche Gruppen I, Springer-Verlag, 1967.

[5] K. Ishikawa, Finite p-groups up to isoclinism, which have only two conjugacy lengths, J. Algebra 220 (1999), no. 1, 333-354.

[6] X. H. Li and J. Q. Zhang, Finite p-groups and centralizers of non-central elements, Comm. Algebra 41 (2013), no. 9, 3267-3276.

[7] M. Y. Xu, L. J. An, and Q. H. Zhang, Finite p-groups all of whose non-abelian proper subgroups are generated by two elements, J. Algebra 319 (2008), no. 9, 3603-3620.

LIHUA ZHANG

Beijing University of Posts and Telecommunications

Beijing 100876, P. R. China

E-mail address: zhlh@bupt.edu.cn

JIAO WANG

Department of Mathematics

ShanXi Normal University

Linfen, Shanxi, 041004 P. R. China

E-mail address: wangjiaotiedan@163.com

HAIPENG QU

Department of Mathematics

Shanxi Normal University

Linfen, Shanxi, 041004 P. R. China

E-mail address: orcawhale@163.com 